\title{
Complete genome sequence of a novel toti-like virus from the plant-pathogenic oomycete Phytophthora cactorum
}

\author{
Anna Poimala ${ }^{1}\left[\right.$ [ Eeva J. Vainio ${ }^{1}$
}

Received: 11 February 2020 / Accepted: 30 March 2020 / Published online: 4 May 2020

(c) The Author(s) 2020

\begin{abstract}
This report describes the complete genome sequence of a double-stranded RNA (dsRNA) virus infecting the oomycetous plant pathogen Phytophthora cactorum. The virus genome consists of a single dsRNA segment of 5699 bp with two open reading frames predicted to overlap with each other and encoding a putative capsid protein of 705 aa and an RNA-dependent RNA polymerase of 779 aa. Sequence comparisons indicated that this virus, designated as "Phytophthora cactorum RNA virus 1" (PcRV1), shares the highest sequence similarity with the unclassified Pythium splendens RNA virus 1 (58\% RdRp aa sequence identity). Phylogenetic analysis revealed that these two oomycete viruses group together with Giardia lamblia virus (GVL; family Totiviridae) and several unclassified toti-like viruses from arthropods, fish and fungi. This is the first report of a toti-like virus in a member of the genus Phytophthora and the first virus characterized in P. cactorum.
\end{abstract}

Phytophthora cactorum (Leb. and Cohn) Schröeter is an oomycetous phytopathogen of the Kingdom Stramenopila that causes root, collar and crown rot as well as foliar and fruit infections on a broad range of hosts, including over 200 species of trees, ornamentals, and fruit crops [5]. On Betula spp., P. cactorum is mostly a problem in nurseries, causing stem lesions on seedlings [13]. Prior to this report, no viruses had been described in $P$. cactorum, whereas a few viruses are known in congeneric host species: alphaendornaviruses infect members of the Phytophthora taxon 'douglasfir' and $P$. ramorum [9], and four dsRNA viruses have been described in the potato late blight pathogen $P$. infestans [2]. Two of these four are not closely related to other known viruses but have some resemblance to members of the family Astroviridae, whereas one is affiliated with a family-level group tentatively called 'fusagraviruses' and one is an unclassified member of the family Narnaviridae. In this study, we report the complete genome sequence of a new toti-like virus infecting $P$. cactorum, designated as "Phytophthora cactorum RNA virus 1" (PcRV1).

\section{Handling Editor: Ioly Kotta-Loizou.}

Anna Poimala

anna.poimala@luke.fi

1 Natural Resources Institute Finland (Luke), Natural

Resources Unit, Forest Health and Biodiversity,

Latokartanonkaari 9, 00790 Helsinki, Finland

\section{Provenance of the virus material}

Phytophthora cactorum isolate BirchT_KT09 was isolated in 2009 from a trunk lesion on silver birch (Betula pendula Roth.) in Denmark by Kirsten Thinggaard, cultivated on $2 \%$ malt extract agar plates, and identified based on its morphology and ribosomal internal transcribed spacer 2 (ITS 2) sequence. Total dsRNA was extracted from $2.5 \mathrm{~g}$ of cultivated mycelia using cellulose affinity chromatography [12]. A 5.7-kb dsRNA element was purified from an agarose gel (Fig. 1A), and cDNA synthesis was conducted using tagged random hexamer priming followed by PCR amplification as described by Márquez et al. [11] except that Maxima H- Reverse Transcriptase and DyNAzyme II DNA polymerase (Thermo Scientific) were used. The amplicons were cloned using a TOPO TA Cloning Kit, (Thermo Scientific) and sequenced by Macrogen Inc., after which specific primers for amplification of the whole sequence were designed (data not shown). The whole sequence was determined based on direct Sanger sequencing of overlapping PCR amplicons $(<1000$ bp with DreamTaq DNA polymerase and $>1000 \mathrm{bp}$ with Phusion High-Fidelity DNA Polymerase, Thermo Scientific), sequencing the products of at least two separate PCR reactions to cover each nucleotide position. The terminal sequences were determined using T4 RNA adapter ligation followed by PCR amplification with specific primers and Phusion High-Fidelity DNA Polymerase (Thermo Scientific). Sequence data were analyzed using Geneious R10 
A

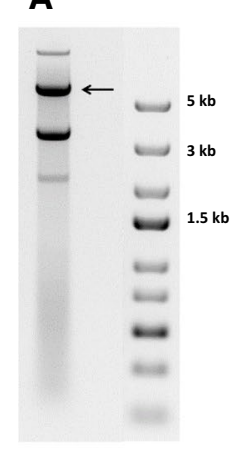

B

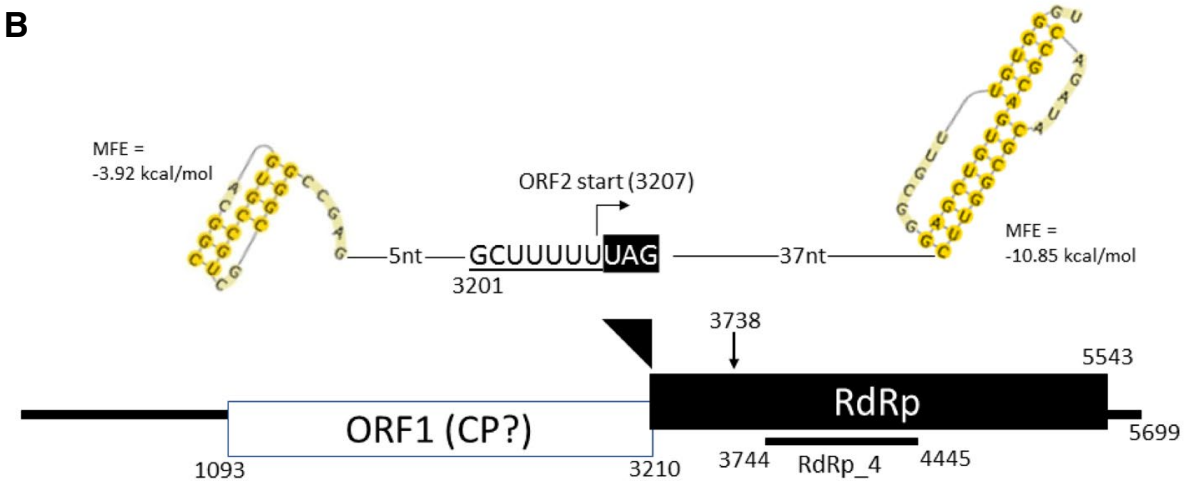

C
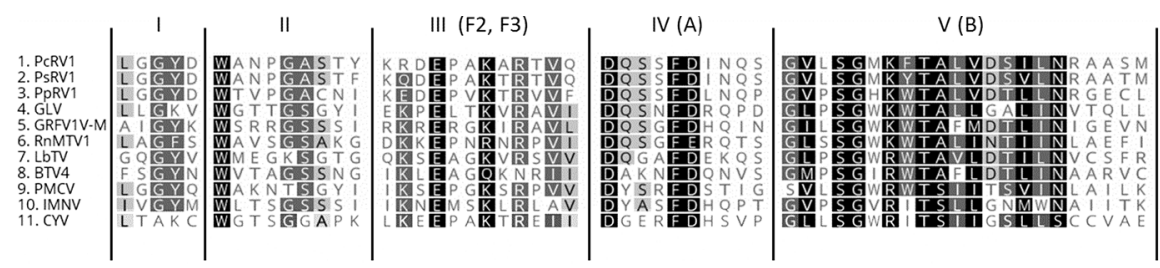

VI (C)

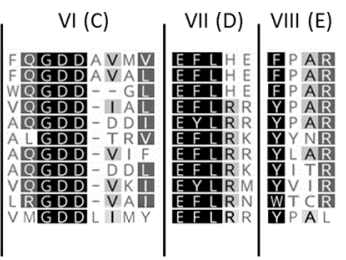

Fig. 1 A. dsRNA profile of Phytophthora cactorum isolate BirchT KT09 visualized in 1\% agarose after 90-min electrophoresis at $120 \mathrm{~V}$. PcRV1 is indicated by an arrow, while the other dsRNA elements present in the same host isolate will be reported elsewhere. The amount of O'GeneRuler Express DNA Ladder (Thermo Scientific) was $0.5 \mu \mathrm{g}$. According to the manufacturer, the $1.5-\mathrm{kb}$ band corresponds to $100 \mathrm{ng}$ of DNA per $0.5 \mathrm{ug}$ of ladder. B. Schematic representation of the genomic organization of PcRV1. The open reading frames (ORFs) and the untranslated regions (UTRs) are indicated by open

(Biomatters Ltd., New Zealand). Sequence alignment for phylogenetic analysis was conducted using MAFFT v7.388 and the Blosum45 substitution matrix. Phylogenetic analysis was carried out using the MrBayes program implemented in Geneious R10 with the following parameters: rate matrix $\mathrm{LG}+G+I$ with five gamma categories; $1.1 \times 10^{6}$ cycles for the MCMC algorithm, sampling one tree per 200 cycles; discarding $10^{5}$ samples as burn-in.

\section{Sequence properties}

The complete genome sequence of PcRV1 (isolate PcRV1_ BpKT09, GenBank accession number MN956531) consists of a single segment of 5,699 bp (Fig. 1A) with a 55\% G+C content. The genome has a 1092-nt-long UTR at the $5^{\prime}$ terminus and a 156-nt UTR at the $3^{\prime}$ terminus (Fig. 1B). The genome is predicted to encode two large ORFs. ORF1 (705 aa; nt positions 1,093-3,210) shares $50.85 \%$ aa BLASTx identity (query coverage, $99 \%$ ) with the putative coat protein of Pythium splendens RNA virus 1 (PsRV1; BBJ21454), as well as $37.80 \%$ and $29.72 \%$ BLASTx identity with putative $\mathrm{CP}$ sequences named as "Plasmopara viticola associated totivirus-like 1 and 2", respectively (unpublished; GenBank bars and single lines, respectively. The underlined sequence shows a putative slippage site (shifty heptamer), and the putative H-type pseudoknots are shown $37 \mathrm{nt}$ to the right and $5 \mathrm{nt}$ to the left. The first AUG of ORF2 is at nt position 3738. C.. Sequence comparison of the eight conserved motifs [1] in the RdRps of PcRV1 and other representative viruses of the family Totiviridae. Colors indicate aa similarity. Black, 100\% similar; dark grey, $80-100 \%$ similar; light grey, 60-80\% similar; white, less than $60 \%$ similar. Virus name abbreviations are the same as in the dendrogram (Fig. 2)

accession numbers MN545914-5). Except for these three viruses, the predicted ORF1 did not share any BLASTp or BLASTx similarity with known proteins, and no putative conserved domains were detected with NCBI conserved domain search.

A shifty heptamer motif (GCUUUUU; nt positions 3,2013,207 ), which may facilitate -1 programmed ribosomal frameshifting in virus transcripts, was observed at the end of ORF1 (Fig. 1A). Based on this, the second ORF is predicted to begin with leucine in the shifty heptamer motif at 3,207 $\mathrm{nt}$, overlapping in the -1 frame with ORF1. ORF2 (779 aa; nt positions 3,207-5,543) codes for a putative RNAdependent RNA polymerase (RdRp) and shares the highest BLASTx identity (58.64\%; query coverage, $99 \%$ ) with PsRV1 RdRp (LC467965). No H-type RNA pseudoknot, which could stimulate frameshifting, was detected next to the slippery sequence, although a pseudoknot was predicted at a 37-nt distance from the ORF1 stop codon using HPknotter [4] (Fig. 1B). The ORF2 sequence includes a conserved region resembling members of the pfam02123 protein family, including RdRps from members of the genera Luteovirus, Totivirus and Rotavirus (aa residues 180-501). The genome organization of PcRV1 corresponds to that found in members of the family Totiviridae [6], where the 5'-proximal 
ORF encodes the CP and the 3'-proximal ORF encodes the RdRp. Based on this, we propose that ORF1 encodes a CP also in PcRV1. However, due to the high level of sequence divergence, the putative $\mathrm{CP}$ was not used for further phylogenetic inference.

Viruses resembling Giardia lamblia virus (GLV), the sole classified member of the genus Giardiavirus, family Totiviridae (ICTV Master Species List 2018b.v2 at https://talk.ictvo nline.org/), have recently been detected in arthropods, fish and fungi $[4,10,14]$. Phylogenetic analysis based on RdRp sequences (Fig. 2) showed that PcRV1 and the Pythium viruses PsRV1 and Pythium polare RNA virus 1 (PpRV1) form a highly supported clade and cluster among the new GLV-like viruses. Interestingly, Shiba et al. [15] detected a virus-like RdRp sequence similar to that of PsRV1 in the transcriptome of Heterosiphonia pulchra, which also included transcripts related to Phytophthora cactorum. Thus, the natural sample of $H$. pulchra might have included an associated $P$. cactorum hosting a similar toti-like virus. The virus-like sequence from $H$. pulchra showed $37.0 \%$

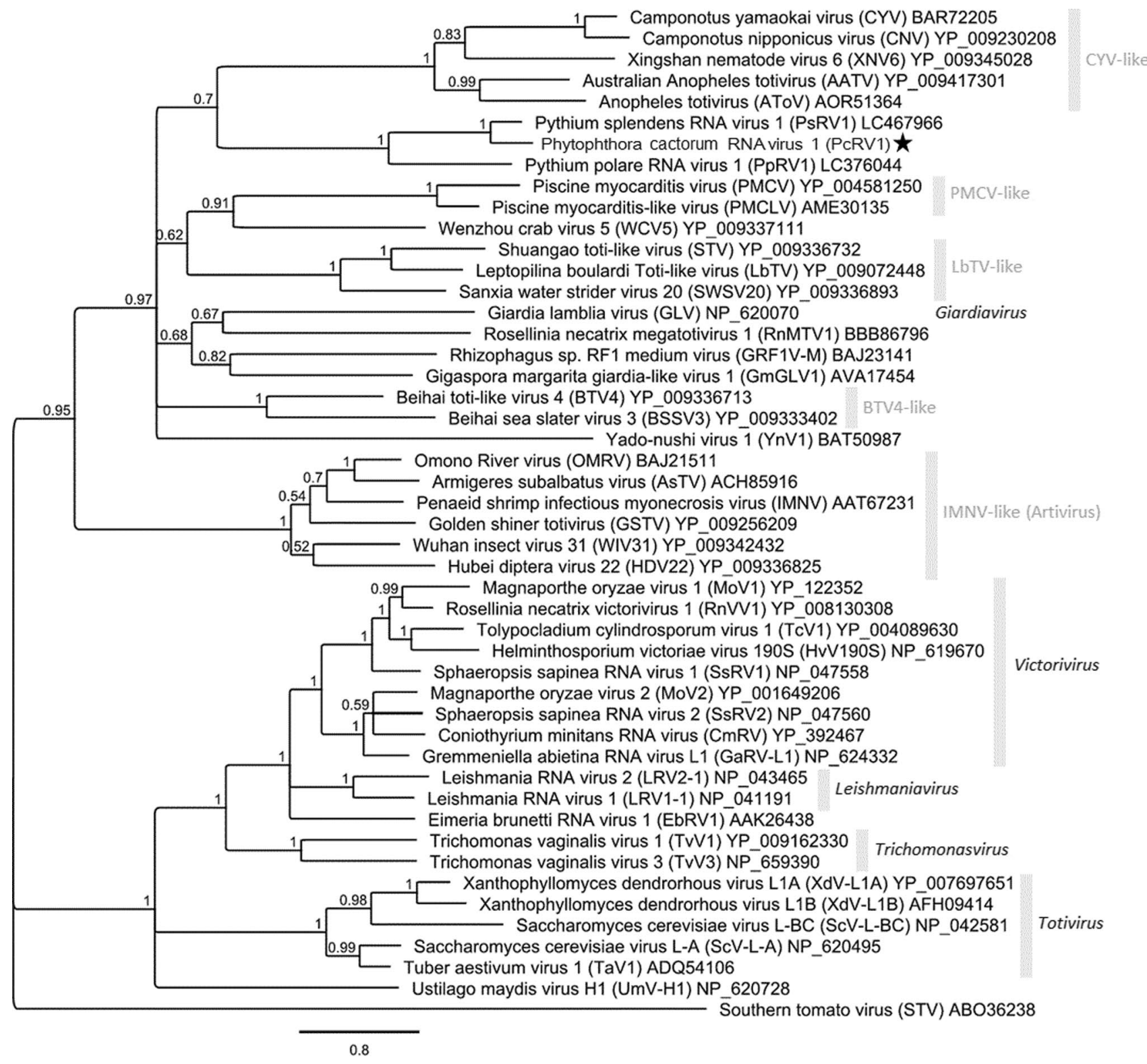

Fig. 2 Phylogenetic analysis based on the RdRp amino acid sequences of PcRV1 and those of related viruses including representatives of the five Totiviridae genera as well as other related phylogenetic groups awaiting classification [14]. The sequence region included in the MAFFT alignment contained the RdRp conserved motifs I-VIII and consisted of 492 aa sites. The analysis was conducted with MrBayes, using southern tomato virus as an outgroup. The scale bar shows 0.8 aa substitutions per site, and percentage posterior probabilities are shown at branch nodes 
aa sequence identity to the PcRV1 RdRp based on global alignment. Among the GLV-like viruses, the presence of a shifty heptamer motif together with the absence of a nearby RNA pseudoknot is also found in piscine myocarditis virus (PMCV) and Camponotus yamaokai virus (CYV) [14]. In addition, Jamal et al. [7] did not detect the CP-RdRp fusion product of Alternaria alternata victorivirus 1 (AalVV1), whose genome also has a similar structure, suggesting that the translation of AalVV1 RdRp might involve proteolytic processing or a novel type of termination-coupled reinitiation mechanism.

According to the virus species demarcation criteria for the genus Totivirus in the family Totiviridae [8], viruses found only in distinct host species are for that reason considered members of different species, and less than $50 \%$ sequence identity at the protein level generally reflects a species difference. The RdRp sequence identity between PcRV1 and the Pythium virus PsRV1 exceeds this threshold but remains moderate (ca. 58\%) and corresponds to the $60 \%$ maximum identity criterion set for different species of the genus Victorivirus in family Totiviridae. In addition, the hosts represent different oomycete genera. Therefore, we propose that these viruses should be considered members of different species. As more related species are described, the ICTV may need to adjust the species demarcation criteria for this virus group accordingly. It should be noted that strains of Pythium and Phytophthora often co-occur in soil and diseased plants, which could provide a potential route for interspecies virus transmission. However, no mechanisms for interspecies virus transmission between members of oomycete genera have been described thus far, and in the case of Phytophthora infestans, even intraspecies transmission of viruses between somatically incompatible isolates has been shown to be unsuccessful [3].

Acknowledgements Open access funding provided by Natural Resources Institute Finland (LUKE). Kirsten Thinggaard is thanked for providing the Phytophthora cactorum isolate. Juha Puranen, Tuija Hytönen and Alex Nordlund are acknowledged for technical assistance, and Suvi Sutela is thanked for valuable discussions regarding virus genome characterization.

This project has received funding from the European Union's Horizon 2020 Research and Innovation Programme under Grant Agreement No. 773567

\section{Compliance with ethical standards}

Conflict of interest The authors declare no conflict of interest.

Ethical approval This article does not contain any studies with human participants or animals performed by any of the authors.

Open Access This article is licensed under a Creative Commons Attribution 4.0 International License, which permits use, sharing, adaptation, distribution and reproduction in any medium or format, as long as you give appropriate credit to the original author(s) and the source, provide a link to the Creative Commons licence, and indicate if changes were made. The images or other third party material in this article are included in the article's Creative Commons licence, unless indicated otherwise in a credit line to the material. If material is not included in the article's Creative Commons licence and your intended use is not permitted by statutory regulation or exceeds the permitted use, you will need to obtain permission directly from the copyright holder. To view a copy of this licence, visit http://creativecommons.org/licenses/by/4.0/.

\section{References}

1. Bruenn JA (2003) A structure and primary sequence comparison of the viral RNA-dependent RNA polymerases. Nucleic Acids Res 31:1821-1829

2. Cai G, Hillman BI (2013) Phytophthora viruses. Adv Virus Res 86:327-350

3. Cai G, Fry WE, Hillman BI (2019) PiRV-2 stimulates sporulation in Phytophthora infestans. Virus Res 271:197674

4. de Lima JGS, Teixeira DG, Freitas TT, Lima JPMS, Lanza DCFR (2019) Evolutionary origin of 2A-like sequences in Totiviridae genomes. Virus Res 259:1-9

5. Erwin DC, Ribeiro OK (1996) Phytophthora diseases worldwide. American Phytopathological Society Press, St. Paul, MN

6. Huang C-H, Lu CL, Chiu H-T (2005) A heuristic approach for detecting RNA H-type pseudoknots. Bioinformatics 21:3501-3508

7. Jamal A, Sato Y, Shahi S, Shamsi W, Kondo H, Suzuki N (2019) Novel victorivirus from a Pakistani isolate of Alternaria alternata lacking a typical translational stop/restart sequence signature. Viruses 11:577

8. King AM, Adams MJ, Lefkowitz EJ, et al. (eds) (2011) Virus taxonomy: IXth report of the International Committee on Taxonomy of Viruses, 9th edn. Elsevier, Waltham

9. Kozlakidis Z, Brown NA, Jamal A, Phoon X, Coutts RHA (2010) Incidence of endornaviruses in Phytophthora taxon douglasfir and Phytophthora ramorum. Virus Genes 40:130-134

10. Liu H, Fu Y, Xie J, Cheng J, Ghabrial SA, Li G, Peng Y, Yi X, Jiang D (2012) Evolutionary genomics of mycovirus-related dsRNA viruses reveals cross-family horizontal gene transfer and evolution of diverse viral lineages. BMC Evol Biol 12:91

11. Márquez LM, Redman RS, Rodriguez RJ, Roossinck MJ (2007) A virus in a fungus in a plant: three-way symbiosis required for thermal tolerance. Science 315:513-515

12. Vainio EJ, Keriö S, Hantula J (2011) Description of a new putative virus infecting the conifer pathogenic fungus Heterobasidion parviporum with resemblance to Heterobasidion annosum P-type partitivirus. Arch Virol 156:79-86

13. Rytkönen A, Lilja A, Petäistö R-L, Hantula J (2008) Irrigation water and stem lesions on Betula pendula in a forest nursery. Scan J For Res 23:404-411

14. Sasai S, Tamura K, Tojo M, Herrero ML, Hoshino T, Ohki ST, Mochizuki T (2018) A novel non-segmented double-stranded RNA virus from an Arctic isolate of Pythium polare. Virology 522:234-243

15. Shiba K, Hatta C, Sasai S, Tojo M, Ohki ST, Mochizuki T (2018) A novel toti-like virus from a plant pathogenic oomycete Globisporangium splendens. Virology 537:165-171

Publisher's Note Springer Nature remains neutral with regard to jurisdictional claims in published maps and institutional affiliations. 